
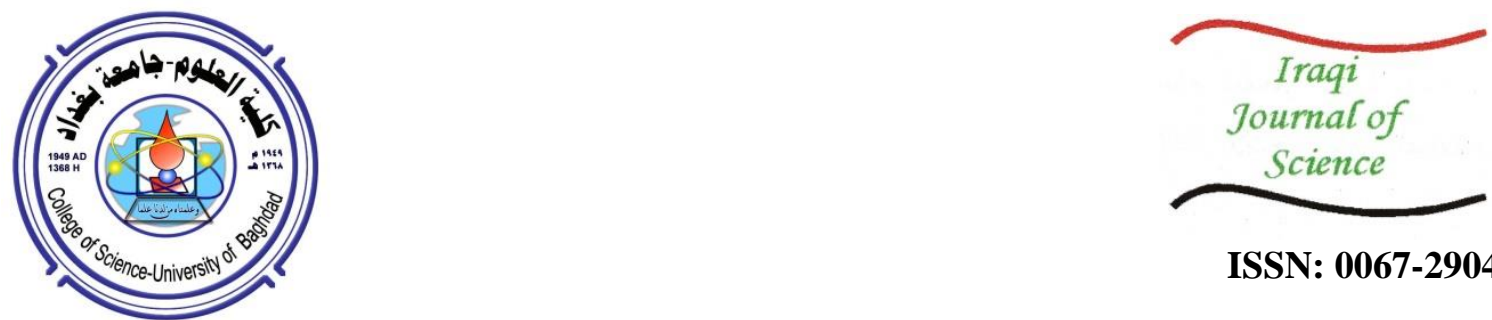

ISSN: 0067-2904

\title{
A Comparison Between Different Susceptibility Test Methods to Evaluate the Antibacterial Activity of Olibanum and Alum Against the "Red Complex" Periodontal Pathogens
}

\author{
Sumaya A. S. M. Al-Hamdoni ${ }^{{ }^{*}}$, Amera M. M. Al-Rawi ${ }^{2}$ \\ ${ }^{1}$ Department of Biophysics, College of Science, University of Mosul, Mosul, Iraq \\ ${ }^{2}$ Department of Biology, College of Science, University of Mosul, Mosul, Iraq
}

Received: 25/2/2020

Accepted: $31 / 3 / 2020$

\begin{abstract}
Testing the sensitivity of periodontal pathogens requires the selection of an easier and more reliable method to be used with such anaerobic bacteria that need a long period of time for growth. Natural materials are a new era of antibacterial agents to control periodontal infections. The aims of the current study were to test the antibacterial activity of two natural agents, namely olibanum and alum, against three types of red complex periodontal pathogens and compare the application of agar diffusion and microdilution methods for testing the susceptibility. Gingival crevicular fluid from pockets with chronic infections was sampled as a source for the three types of bacteria, Porphyromonas gingivalis, Tannerella forsythia and Treponema denticola (the red complex pathogens). The samples $(\mathrm{n}=30)$ were cultured on three types of media, namely Schaedler Anaerobe Agar, Tannerella forsythia (TF) agar, and Trypton Yeast extract Gelatin Volatile fatty acids and Serum (TYGVS) agar for the three types of red complex pathogens. After anaerobic growth, the isolates of red complex pathogens were identified by cultural and cellular morphological characteristics and confirmed by molecular diagnosis. The antibacterial activity of the two natural materials was tested by agar (disk and well) diffusion and microdilution method (spectrophotometer- and resazurin- based). Ciprofloxacin (CIP) and chlorhexidine (CHX) were used as controls. Minimal Inhibitory Concentrations (MICs) of the four reagents (olibanum, alum, CIP, and CHX) were determined on the three types of bacteria. MIC values by each susceptibility method were compared and analyzed statistically at $p$-value $\leq 0.05$. The results showed that resazurin- based microdilution method was the easiest and simplest approach which gave reliable reads. MIC values of the four regents differed from a method to another and from one bacterium to another. Statistically, there were no significant values among these differences, except for olibanum which was statistically more significant than the other reagents.
\end{abstract}

Keywords: antibacterial activity; susceptibility test of anaerobes; natural materials.

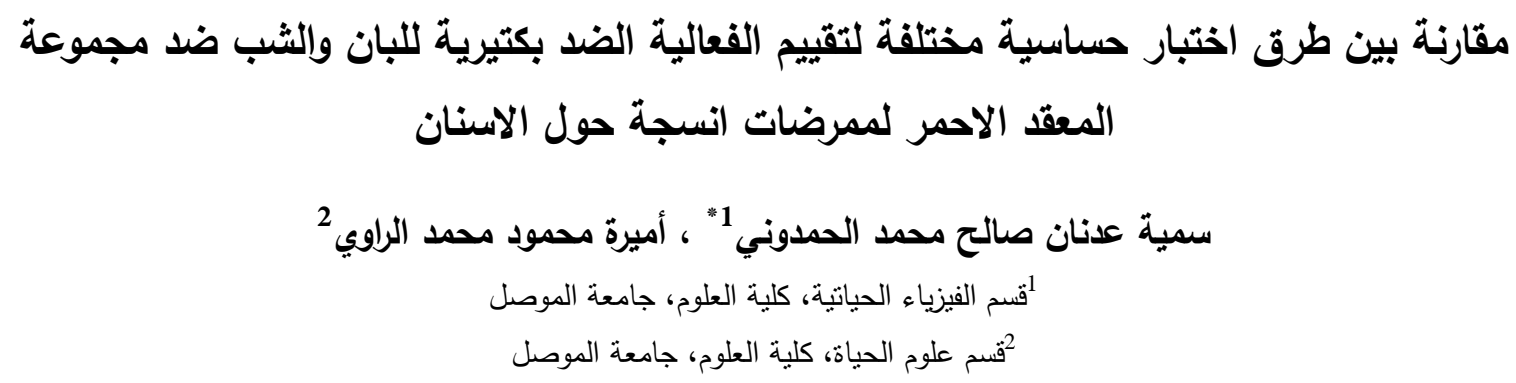

*Email: zkhaldon@yahoo.com 


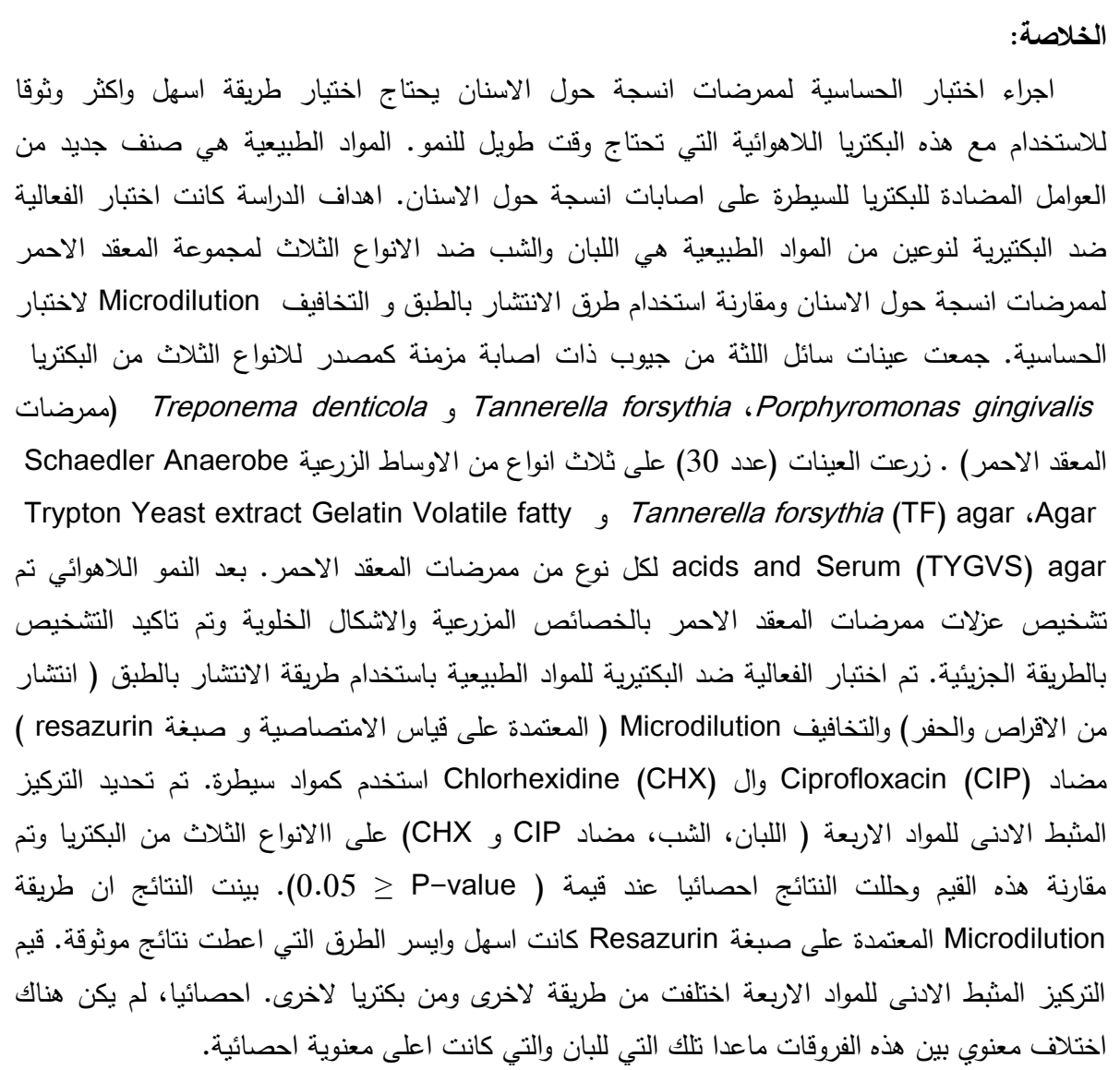

\section{Introduction}

Screening the activity of crude extracts or purified compounds form natural substances requires the employment of a simple, rapid, reliable, sensitive and cost- effective in vitro assay. Choosing the correct method is important for generating high-quality data of the new antimicrobial compounds and extracts [1]. Anaerobic susceptibility testing (AST) methods standardized by the Clinical and Laboratory Standards Institute (CLSI) include agar dilution, broth microdilution, and E- test [2]. However, these routine methods when applied for anaerobes are time- consuming and expensive, especially if one need to screen several agents with several microbial types [3]. In addition to, standard diameters of the inhibition zone are available for comparing the results of only the tests for standard antibiotics, but there are no standard explanations for comparing the results of the tests of natural materials [4].

Resazurin is the most commonly used redox indicator in media culturing anaerobes to monitor the redox potential because it is generally non-toxic to bacteria and effective at very low concentrations [5]. This indicator is dark blue in its inactive form that can penetrate the cells and is irreversibly reduced to the fluorescent pink resofurin by cellular oxidoreductase. In a second reversible reduction step, the colorless hydroresorufin is formed. The resorufin/hydroresorufin redox couple becomes totally colorless at high redox potential and regains a pink color at a lower redox potential. Resofurin is soluble and diffuses passively into the surrounding medium enabling an easy visual reading of the results. The intensity of the fluorescence is proportional to the number of living metabolic active cells. Therefore, resazurin reduction provides indications about cell viability, a benefit which can be utilized in testing bacterial susceptibility $[3,6]$.

Mechanical removal of subgingival plaque is the first line in periodontal treatment. However, not all patients respond favorably and hence antibiotic therapy is recommended to improve the treatment outcome [7]. Systemic antibiotics are of a particular benefit as they enter the periodontal tissues via serum to suppress periodontal pathogens [8]. Plant- derived antimicrobials carry prospective agents as natural antimicrobials with chemotherapeutic benefits that are powerful systems for the treatment of 
infectious diseases $[9,10]$. These natural products are environmentally safe, easily available and cheap compared to the toxicity and side effects of allopathic medicines [11, 12].

Boswellia sp. is a deciduous tree that involves about 20 species. The exuded gum from the tree appears as milk- like resin which hardens to orange-brown gum resin known as frankincense or olibanum. It has been utilized as a fixative in perfumes, soaps, lotions and in medicine for its antiseptic, anti-inflammatory, anti-bacterial, anti-fungal and anti-cancer activities [9, 13]. Alum is the crystalized double sulphates aluminum salt with the formula $\mathrm{KAl}\left(\mathrm{SO}_{4}\right)_{2} \cdot 12 \mathrm{H}_{2} \mathrm{O}$. It is soluble in water and has been used for the improvement and preservation of foods and cosmetics and as a flocculants in water treatments [11]. It has been recommended by the Food and Drug Administration (FDA) for the topical treatment of burns, ulcers and abscess or as a deodorant and astringent agent. It has a bacteriostatic action on cell surfaces with very low permeability into cells and thus little chance of systemic absorption [14].

Porphyromonas gingivalis, Treponema denticola and Tannerella forsythia or the "red complex" group, are known as the most responsible and virulent pathogens associated with the chronic infections of periodontitis [15]. Therefore, the current study selected these three bacteria as representative types of periodontopathogens and aimed to explore the effectiveness of olibanum (Boswellia sp.) and alum as natural materials to control the growth of these periodontal bacteria. We also aimed to find out the easier and more rapid and accurate method for testing the sensitivity of such anaerobic bacteria to antibacterials.

\section{Materials and methods}

Bacterial source: Paper points sized \#40 were used to sampling gingival crevicular fluids from patients suffering from chronic periodontitis as a source of the three types of bacteria. The samples were collected from pockets that have a depth of $\geq 4 \mathrm{~mm}$ and positive bleeding on probe and put in phosphate buffered saline (PBS) until reaching the lab. Specialized dentists helped in the diagnosis and sampling.

Bacterial culturing and identification: In the Microbiology lab/College of Science, the samples were cultured by streaking into three types of culture media; Schaedler Anaerobe Agar with blood, haemin, vitamin K and vancomycin was used for the growing of Porphyromonas gingivalis [16] and Tannerella forsythia. (TF) agar for Tannerella forsythia [17], and TYGVS agar for Treponema denticola [5]. All plates were incubated in anaerobic jars using CampyGen, microaerophilic generation system (Oxiod Ltd, Japan) for 4 days at $37^{\circ} \mathrm{C}$. After that, bacterial colonies corresponding to the three types of red complex pathogens were identified by morphological characteristics of growth, microscopic examination with cell morphology and gram stain, and by the molecular Loop Mediated Isothermal Amplification (LAMP) method.

Method of LAMP technique: Set of four primers targeted to 16SrDNA gene was added to the extracted DNA. WarmStart Colorimetric LAMP 2X Master Mix was used which contained Bst DNA polymerase and the $\mathrm{pH}$ indicator, phenol red. The reaction mixture was set at $65^{\circ} \mathrm{C}$ for $30 \mathrm{~min}$., and then the change of color of the reaction mixture from pink to yellow was observed.

Natural materials and standard antimicrobials: Two natural materials, namely olibanum (Boswellia sp.) and alum (potassium alum) (both purchased from local a perfumer market in Mosul city) and two synthetic drugs, namely ciprofloxacin (CIPLA LTD, India) and chlorhexidine digluconate (Scitra Co., UAE) were tested for their antibacterial effects on the three types of red complex pathogens for growth inhibition.

Effects of natural materials on bacterial growth: The antibacterial effects were studied in terms of minimal inhibitory concentration (MIC) on the growth of the three types of red complex bacteria. Two methods were compared for their reliability to display the MIC values of the antimicrobial agents, agar diffusion (disk diffusion and well diffusion) and 96-well plate microdilution (spectrophotometer assay and resazurin- based assay). Ciprofloxacin (CIP) and Chlorhexidine digluconate (CHX) were used as a positive control.

Preparation of suspension required for agar diffusion assay: Olibanum and alum were grinded to prepare stock solutions at $0.5 \mathrm{~g} / \mathrm{ml}$ for olibanum and $0.6 \mathrm{~g} / \mathrm{ml}$ for alum in warmed distilled water $\left(80^{\circ} \mathrm{C}\right)$ and dissolved by stirring. Liqueur extract of olibanum was obtained after overnight soak in warm water. Stock solutions of $200 \mathrm{mg} / 100 \mathrm{ml} \mathrm{CIP}$ and $0.12 \mathrm{w} / \mathrm{v} \%$ CHX (equal to $120 \mathrm{mg} / 100 \mathrm{ml}$ [18]) were used. Two- fold dilutions were made from each stock solution in sterilized distilled water. 
Bacterial suspension in normal saline, equivalent to McFarland 0.5, was prepared from each bacterial type.

Disk diffusion method: This method was performed as previously indicated [19] as follows: Each bacterial suspension was spread on its corresponding agar plate. Sterilized filter discs $(6 \mathrm{~mm})$ saturated with $15 \mu$ of different concentrations of each reagent were distributed on the surface of an inoculated plate. After 4 days of anaerobic incubation, the inhibition zones were measured to point the MIC value and photographed.

Well diffusion method: This method was performed according to a previous publication [5] as follows: Each bacterial suspension was spread on its corresponding agar plate. Wells with $6 \mathrm{~mm}$ diameter were made and filled with $50 \mu$ l of the tested agent. After 4 days of anaerobic incubation, the inhibition zones were measured.

96-Well plate microdilution and Resazurin- based assay: As formerly designated [4], two sets of plates, one for spectrophotometer assay and the other for resazurin assay, were prepared as follows: A stock solution of each reagent was prepared in a broth medium of each bacterial type at $50 \%(\mathrm{w} / \mathrm{v})$ for

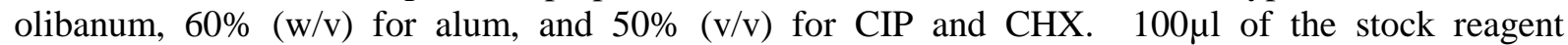
containing- broth was added to column 1 of the 96-well plate. Row A was assigned for olibanum, B for alum, $\mathrm{C}$ for $\mathrm{CHX}$, and D for CIP. From the reagent free- broth medium, $50 \mu 1$ was added to columns 2 till 11 , whereas $100 \mu$ l was added to column 12 as a negative control to monitor sterility. The stock of the reagent containing- broth was two-fold diluted by serial transferring of $50 \mu \mathrm{l}$ from

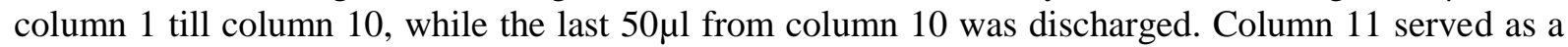
positive control for bacterial growth. $50 \mu 1$ of the bacterial suspension in normal saline (equivalent to McFarland 0.5) was added to each well of columns 1-11. The plates were covered and incubated anaerobically for $72 \mathrm{hr}$. for the spectrophotometic assay and for $30 \mathrm{hr}$. for the resazurin- based assay. For the spectrophotometic assay, the absorbance of the growth was measured by a microplate reader at an optical density (OD) of $630 \mathrm{~nm}$ and the reads versus reagents' concentrations were plotted in a simple linear regression modeling. For the resazurin- based assay, $30 \mu \mathrm{l}$ of resazurin solution was added to each well of columns 1-12 and further incubated for $4 \mathrm{hr}$. The results were read as a change of resazurin color from blue/purple to pink- colorless or no change. The results were finally photographed.

Statistical analysis: Statistical comparison of the results with both negative and positive controls was performed using the one sample $t$ - test to approve the significant effects of each reagent within each type of bacteria. Statistical comparisons of the effects of each reagent among all bacterial types were achieved using ANOVA test. Statistical comparisons among the effects of all reagents on each bacterial type were performed using ANOVA test. The significance was considered at $P$-value $\leq 0.05$.

\section{Results and Discussion}

Identification of bacterial isolates: Colonies which were circular, convex with entire- edge black pigmented and beta-hemolysis on Schaedler Anaerobe Agar (Figure- 1A) and gram negative coccobacilli cells were selected to be $P$. gingivalis. On Tannerella forsythia (TF) agar, colonies which were tiny white Figure-(1B) with gram negative bacilli were selected to be T. forsythia. White- hazy or creamy colonies on TYGVS agar Figure-(1C) with gram negative spirals were selected to be $T$. denticola. All isolates were confirmed by LAMP molecular method in which the presence of the species specific amplicon was assigned upon reaction mixture color change from pink to yellow of phenol red indicator as a result of the proton accumulation in the positive amplification (Figure-2). 


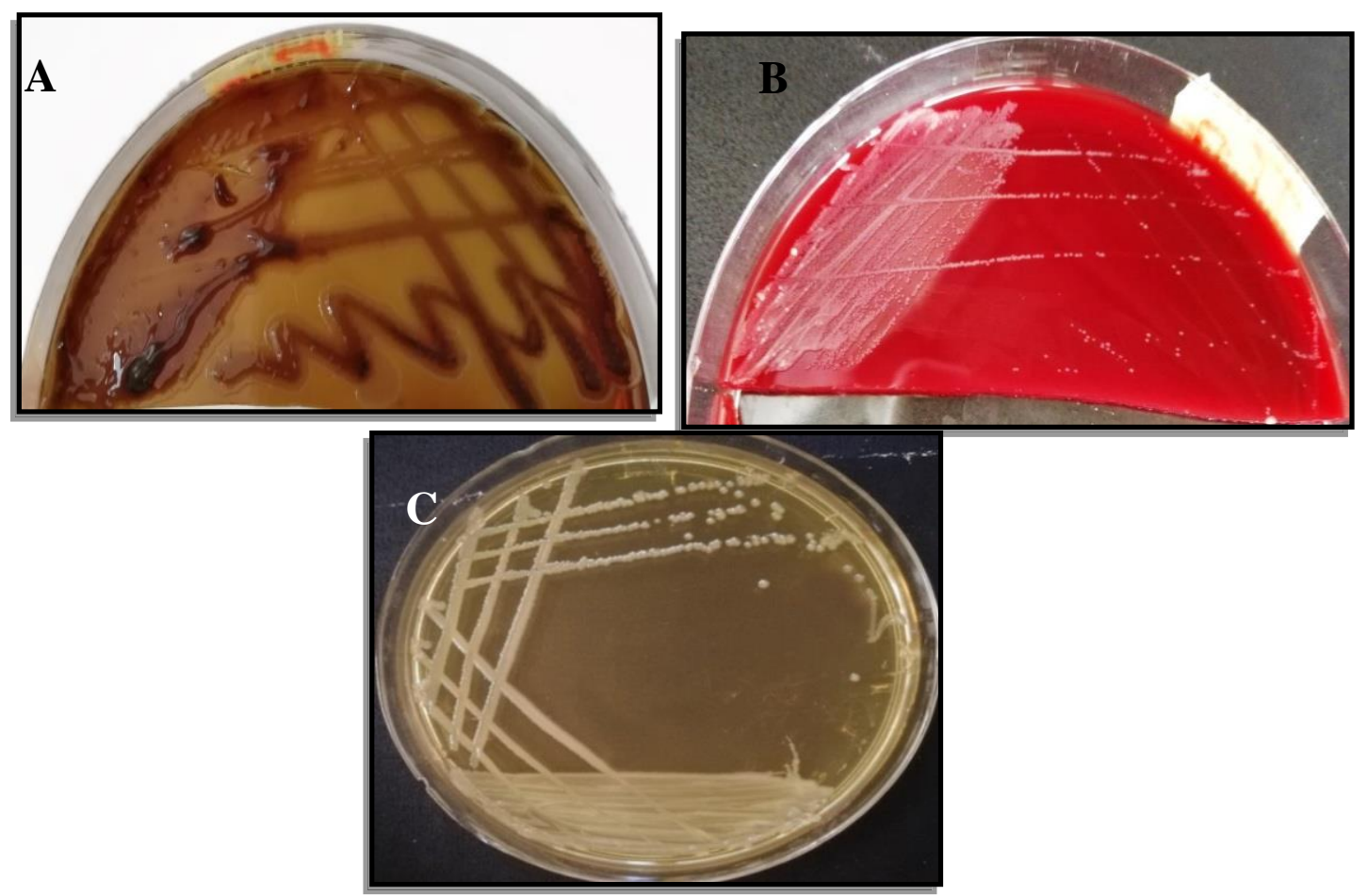

Figure 1-Growth of red complex pathogens on their corresponding medium: (A) P. gingivalis, (B) $T$. forsythia, and (C) T. denticola.

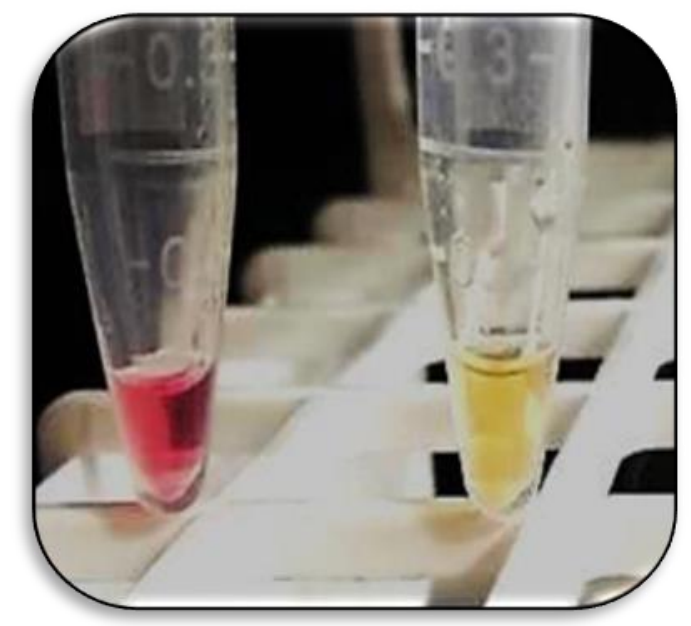

Figure 2-LAMP reaction. Solution with pink color is the negative control; yellow color is the positive amplification of the target DNA.

Antibacterial activities of the four reagents on the growth of red complex pathogens: In the current study, the two natural compounds, olibanum and alum, showed antibacterial activities by inhibiting the growth of the three periodontal bacteria and their MIC values were compared by both agar diffusion and microdilution methods. CIP, a systematic drug and CHX, a local drug, were used as positive controls for inhibition. The stock solutions were $0.5 \mathrm{~g} / \mathrm{ml}$ olibanum, $0.6 \mathrm{~g} / \mathrm{ml} \mathrm{alum,} 120 \mathrm{mg} /$ $100 \mathrm{ml} \mathrm{CHX}$, and $200 \mathrm{mg} / 100 \mathrm{ml}$ for CIP; then they were tow fold diluted. The details of the results are described below.

Comparisons among the susceptibility test methods: Following the agar diffusion method, the inhibitory activity of the tested materials was indicated by the inhibition zone (IZ) of the bacterial growth on the agar medium. The IZs of the concerned concentrations from all tested materials formed by the well- diffusion method were greater than those formed by disk- diffusion against the three types of bacteria, as evidenced in Figures- 3-5. 

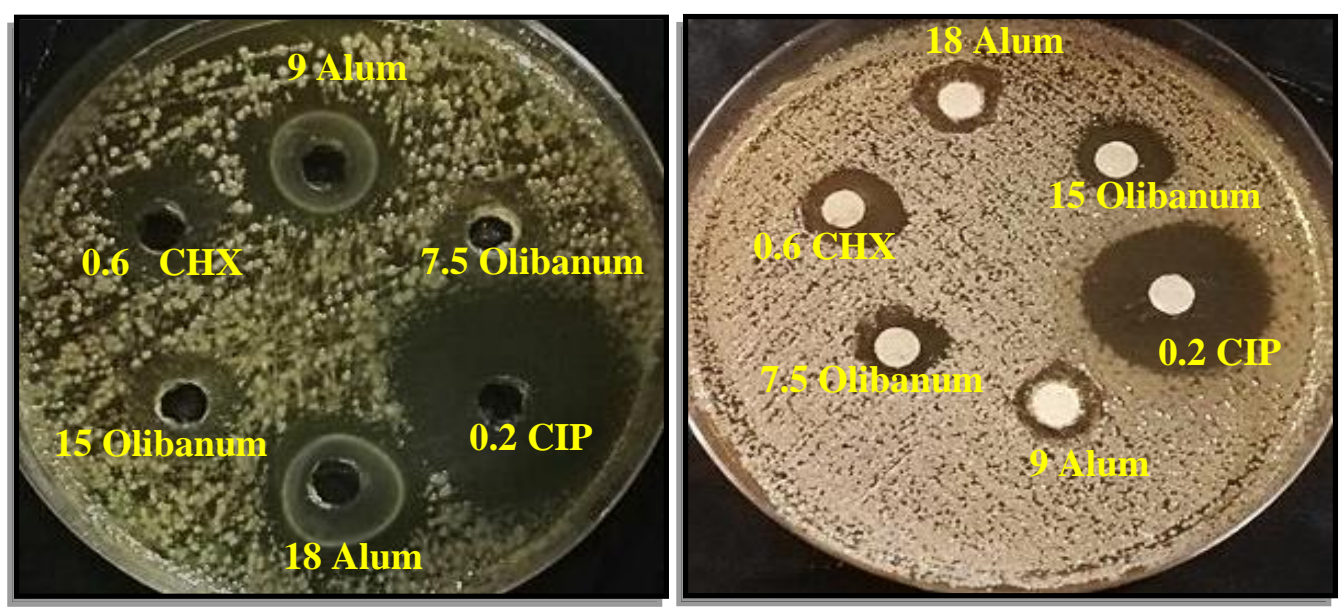

Figure 3-Agar diffusion method for susceptibility of P.gingivalis for antimicrobials with pointed concentrations in $\mathrm{mg} / \mathrm{ml}$.
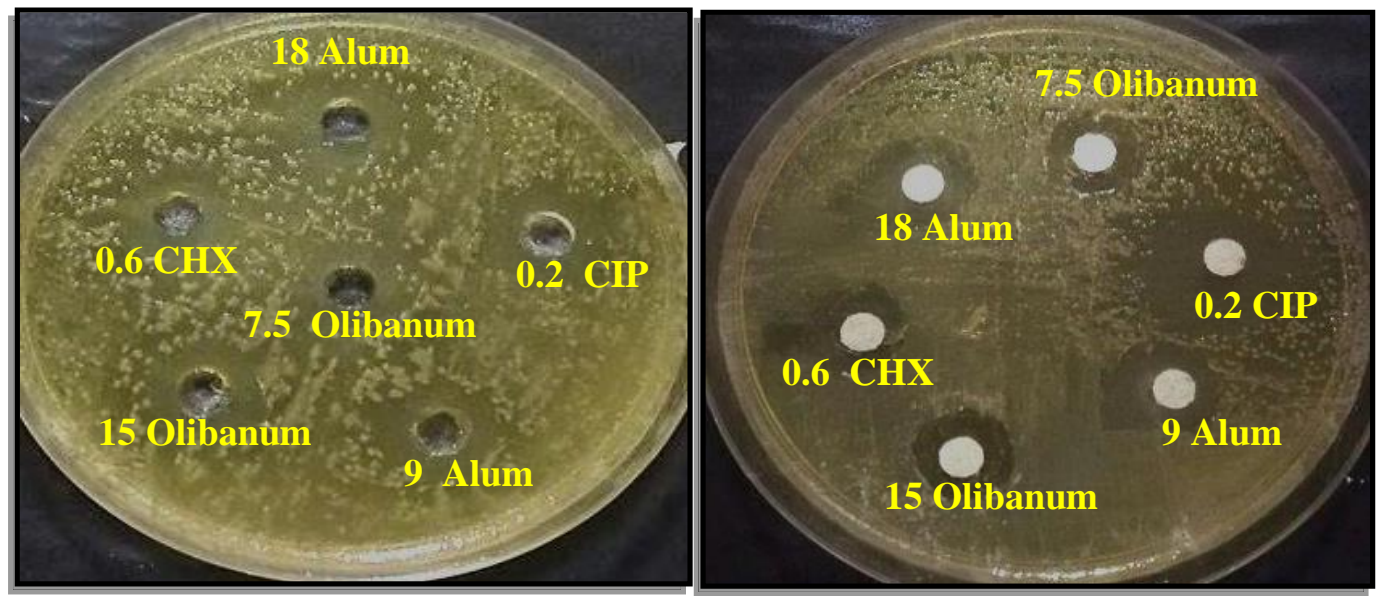

Figure 4-Agar diffusion method for susceptibility of T.forsythia for antimicrobials with pointed concentrations in $\mathrm{mg} / \mathrm{ml}$.
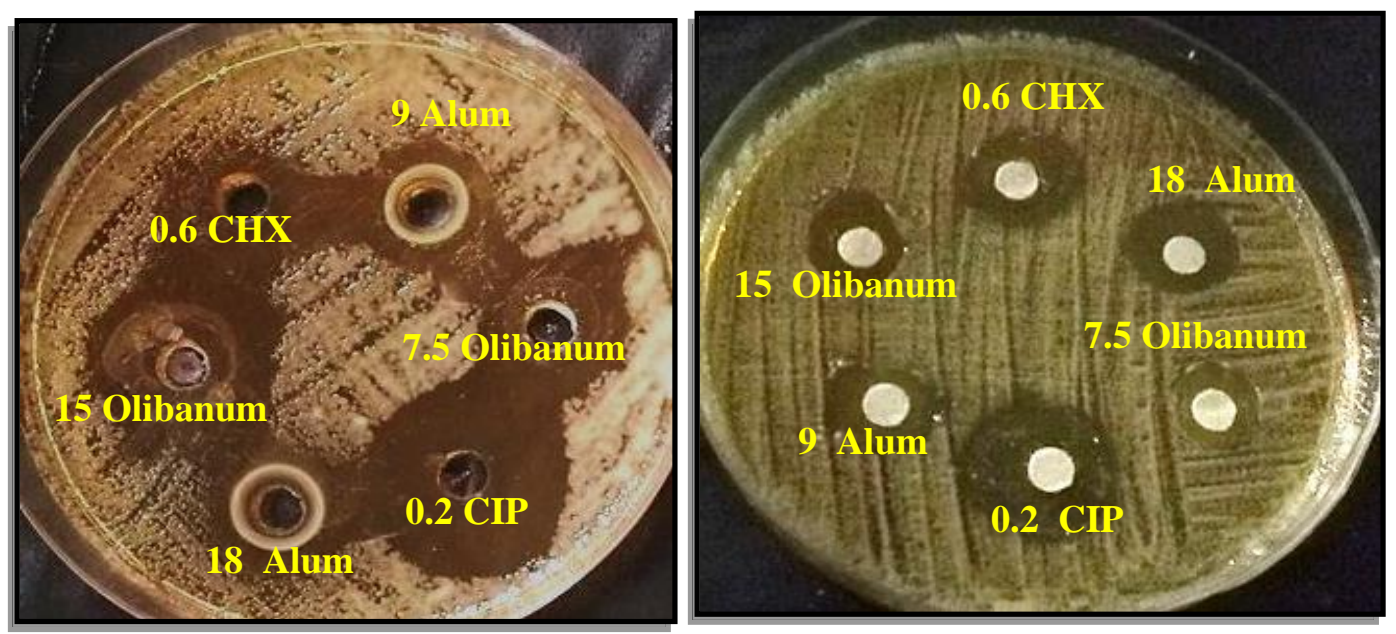

Figure 5-Agar diffusion method for susceptibility of T.denticola for antimicrobials with pointed concentrations in $\mathrm{mg} / \mathrm{ml}$.

Also, the MIC concentrations displayed IZ values by well- diffusion method were lower than those of the disk- diffusion method. For instance, the MICs of olibanum which resulted in IZs of 8-9mm by disk - and well diffusion were 3.7- 4 and 2.4- $3.7 \mathrm{mg} / \mathrm{ml}$, respectively. Alum's MICs which produced IZs of $7-8 \mathrm{~mm}$ were 5.4 and $4.6 \mathrm{mg} / \mathrm{ml}$ by the two methods, respectively. CHX's MICs which produced IZs of 7-8 $\mathrm{mm}$ were $0.15-0.17$ and $0.07-0.15 \mathrm{mg} / \mathrm{ml}$ by the two methods, respectively. 
CIP's MICs which caused IZs of $8-9 \mathrm{~mm}$ were $0.007-0.018$ and $0.006-0.015 \mathrm{mg} / \mathrm{ml}$ by the two methods, respectively. These results, which were the average of five independent experiments, are clarified in Table- 1 . In the well- diffusion method, the bacterial cells were exposed to a larger volume $(50 \mu \mathrm{l})$ of the anti- material than that in the disk diffusion method $(15 \mu \mathrm{l})$. Thus, the IZ was more obvious and larger using the well- diffusion method.

Table 1-MIC and IZs values of the antimicrobial agents against red complex pathogens by the agar diffusion method

\begin{tabular}{|c|c|c|c|c|c|c|c|c|c|c|c|c|}
\hline Agent & \multicolumn{3}{|c|}{ Olibanum } & \multicolumn{3}{|c|}{ Alum } & \multicolumn{3}{|c|}{$\mathrm{CHX}$} & \multicolumn{3}{|c|}{ CIP } \\
\hline & MIC & $\mathrm{mg} / \mathrm{ml})$ & $\mathrm{IZ}^{* * *}$ & MIC & $\mathrm{hg} / \mathrm{ml})$ & $\mathrm{IZ}^{* * * *}$ & MIC & $\mathrm{hg} / \mathrm{ml})$ & $\mathrm{IZ}^{* * * *}$ & $\mathrm{MIC}$ & $\mathrm{g} / \mathrm{ml})$ & $\mathrm{IZ}^{* * * *}$ \\
\hline & disk* & well** & $(\mathrm{mm})$ & disk* $^{*}$ & well** & $(\mathrm{mm})$ & disk* & well** & $(\mathrm{mm})$ & disk* & well** & $(\mathrm{mm})$ \\
\hline P.gingivalis & 4 & 3.7 & 8 & 5.4 & 4.6 & 7 & 0.17 & 0.15 & 7 & 0.018 & 0.015 & 8 \\
\hline T.denticola & 3.7 & 2.4 & 9 & 5.4 & 4.6 & 8 & 0.15 & 0.07 & 8 & 0.007 & 0.006 & 9 \\
\hline T.forsythia & 4 & 3.7 & 8 & 5.4 & 4.6 & 7 & 0.17 & 0.15 & 7 & 0.018 & 0.015 & 8 \\
\hline
\end{tabular}

*: agar disk diffusion, $* *$ : agar well diffusion, $* * *$ : inhibition zone.

In the spectrophotometer- based microdilution method, the absorbance readings of the growth were represented as a correlation between bacterial growth and reagent's concentrations in a simple linear regression modeling. In addition, the inhibition was proved by the linear negative correlation between the growth and reagent's concentration and the negative value of the regression coefficient in the equation, as is depicted in Figure-6. The MIC values were determined as the lowest concentration which resulted in the negative correlation with the growth. The average of three independent results is demonstrated in Table-2.

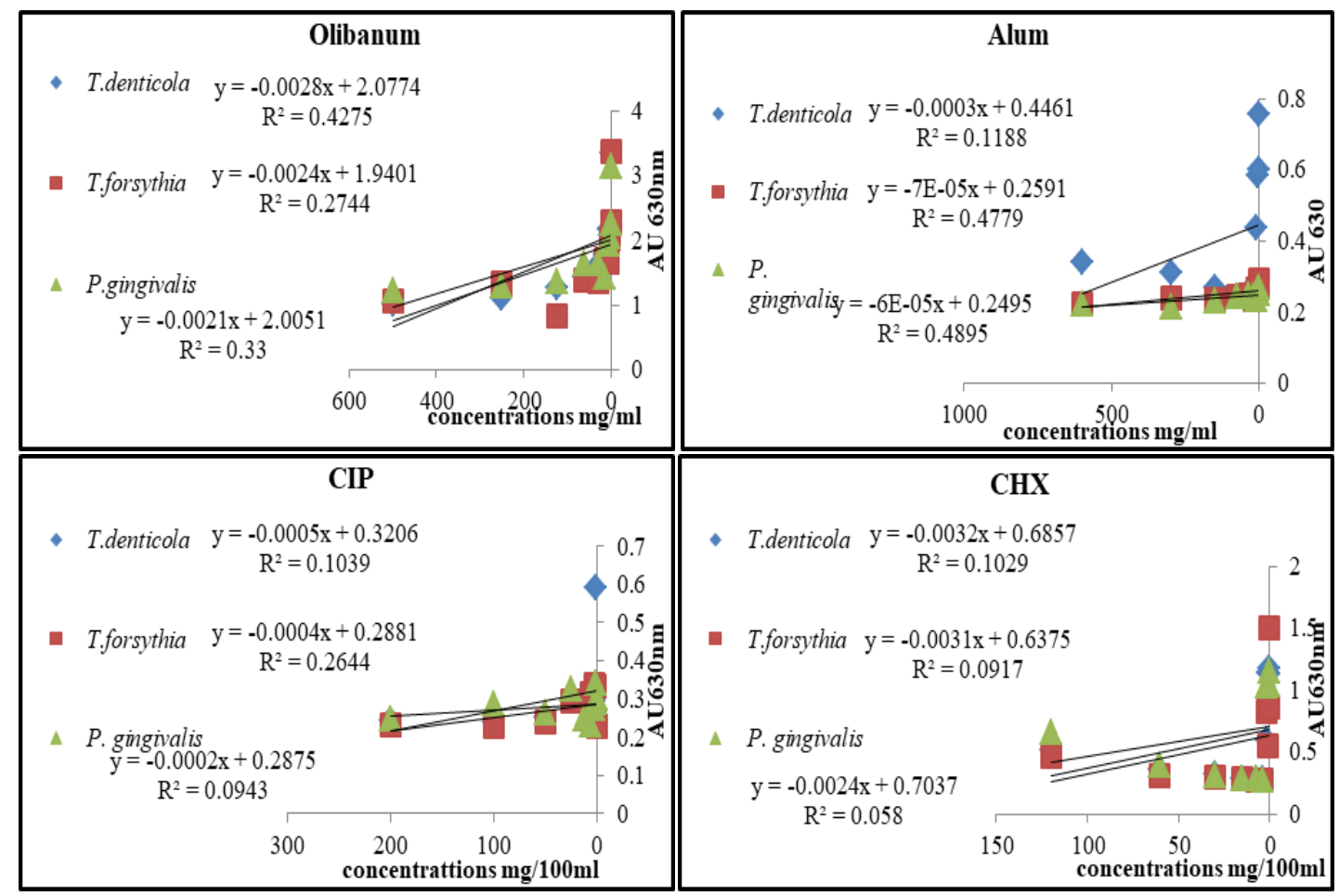

Figure 6-Linear regression modeling showing the effects of the tested reagents on red complex species. 
Table 2-MIC values of the antibacterial agents against red complex species by the spectrophotometerbased microdilution method.

\begin{tabular}{|c|c|c|c|c|}
\hline \multirow{3}{*}{ Bacteria } & \multicolumn{5}{|c|}{ Agent } \\
\cline { 2 - 6 } & $\begin{array}{c}\text { Olibanum (MIC } \\
\mathrm{mg} / \mathrm{ml})\end{array}$ & $\begin{array}{c}\text { Alum (MIC } \\
\mathrm{mg} / \mathrm{ml})\end{array}$ & $\begin{array}{c}\mathrm{CHX}(\mathrm{MIC} \\
\mathrm{mg} / \mathrm{ml})\end{array}$ & $\begin{array}{c}\text { CIP } \\
\mathrm{mg} / \mathrm{ml})\end{array}$ \\
\hline P.gingivalis & 1.8 & 2.3 & 0.04 & 0.0039 \\
\hline T.denticola & 1.5 & 1.9 & 0.037 & 0.0039 \\
\hline T.forsythia & 1.5 & 1.7 & 0.037 & 0.0039 \\
\hline
\end{tabular}

By using resazurin dye in the microdilution method, the growth inhibition was denoted by the remaining of the dye in purple color, while the MIC values were determined in the well of the row with purple resazurin after which the color began to turn into pink or colorless. These results on the types of red complex pathogens are apparent in Figure-7.

\section{T.forsythia}

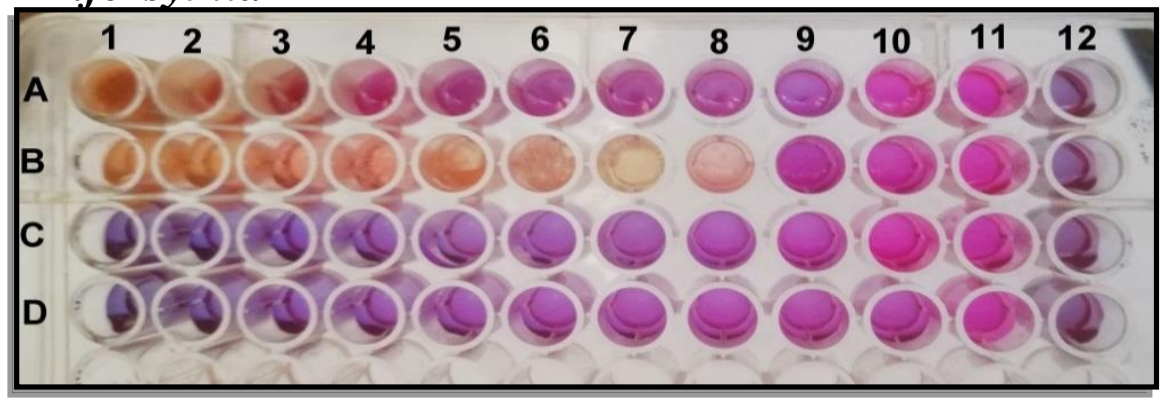

\section{T.denticola}

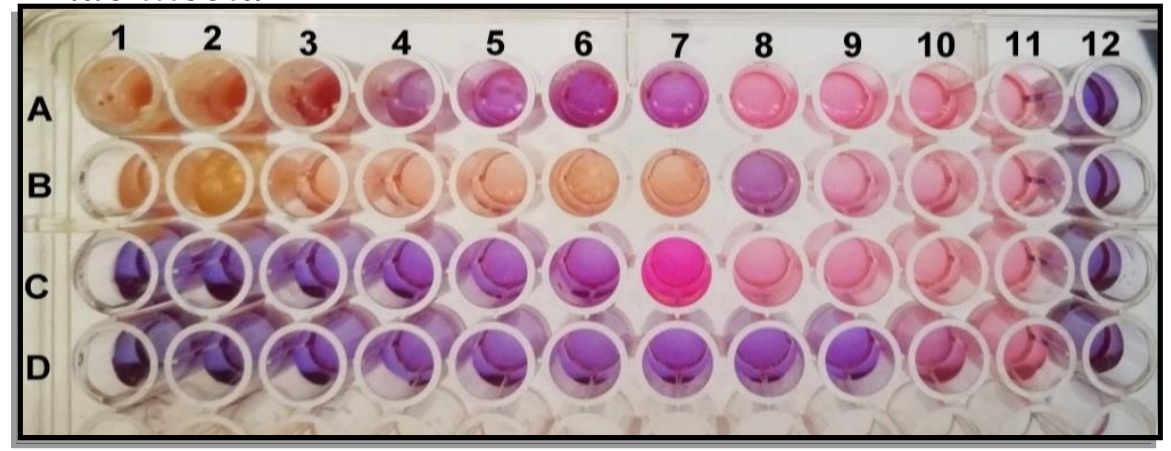

\section{P.gingivalis}

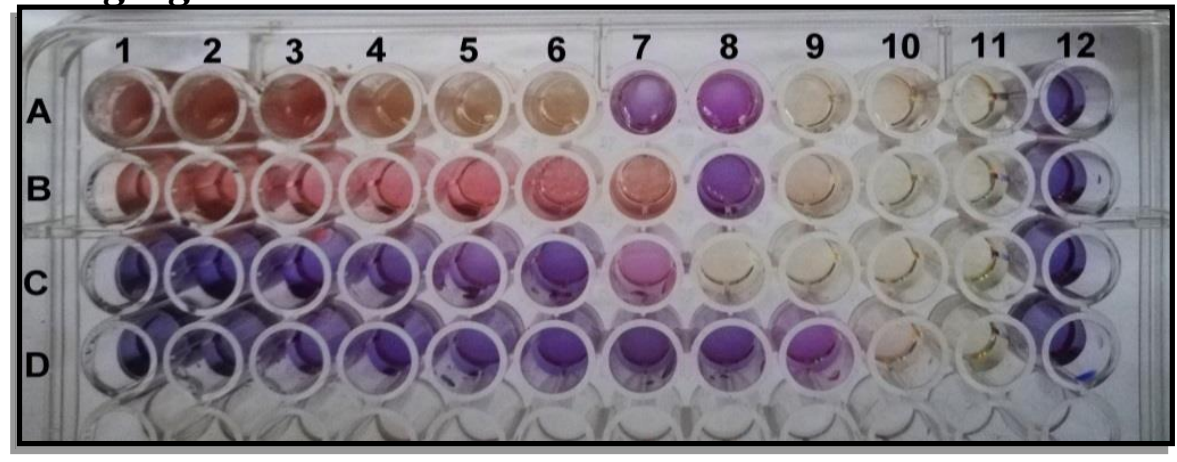

Figure 7- Resazurin-based microplate method; columns 1: double strength of reagents 2-10: tow fold dilution, 11- 12 positive and negative controls, respectively. Rows A: olibanum, B: alum, C: CHX and D: CIP.

The average MIC values of three independent experiments recorded in the resazurin- based microdilution method were 1.4- 1.8, 1.7- 2.3, $0.037-0.04$ and $0.0039 \mathrm{mg} / \mathrm{ml}$ for olibanum, alum, CHX 
and CIP, respectively, as listed in Table-3. The results were closely similar to those observed above using the spectrophotometer- based microdilution method.

Table 3-MIC values of antibacterial agents against red complex species by the resazurin- based method.

\begin{tabular}{|c|c|c|c|c|}
\hline \multirow{3}{*}{ Bacteria } & \multicolumn{5}{|c|}{ Agent } \\
\cline { 2 - 6 } & $\begin{array}{c}\text { Olibanum (MIC } \\
\mathrm{mg} / \mathrm{ml})\end{array}$ & $\begin{array}{c}\text { Alum (MIC } \\
\mathrm{mg} / \mathrm{ml})\end{array}$ & $\begin{array}{c}\text { CHX } \\
\mathrm{mg} / \mathrm{ml})\end{array}$ & (MIC \\
\hline P.gingivalis & 1.8 & 2.3 & 0.04 & 0.0039 \\
\hline T.denticola & 1.4 & 1.7 & 0.04 & 0.0039 \\
\hline T.forsythia & 1.4 & 1.7 & 0.037 & 0.0039 \\
\hline
\end{tabular}

In both methods of microdilution the antibacterial substances were well distributed in the liquid medium. Thus, the bacteria were exposed to the same volume and concentration of the antibacterial agents. This resulted in converging the values of MIC in the two procedures. It was also clear that the MIC values recorded by the microdilution methods were lower than those recorded by the agar diffusion methods.

All methods followed in this study are standard methods that give accurate dependable results in testing the inhibitory effects of the antimicrobials, especially the licensed antibiotics. Variations in the MICs may be due to differences in the media, concentrations of the prepared materials, inoculum, or the endpoint reading method [2]. According to the current study, there was a slight difference between the MIC values of disk diffusion and well diffusion; the inhibition zone formed by the well- diffusion agar method was larger than that formed by the disk-diffusion method, at the same concentration for all agents, as larger amounts were added in the well; thus, the effect of each specific concentration appeared to be stronger using the well- diffusion method (Figures- 3- 5). MIC values of both methods of microdilution were more similar to each other, while MIC values of agar diffusion were larger than those of microdilution. The differences in MIC values of these methods can be attributed to the fact that the ability of the materials to diffuse in agar medium is less than that in broth, which can influence the end result of IZ and MIC. This was evident as MIC values of the microdilution methods were less than those in agar diffusion, especially for the aqueous extracts of the natural materials, while they were easily diffused in liquid media. This attribution was also valid for the agar diffusion method where the materials were easily diffused in the medium of $T$. denticola containing lower agar percentage (7gr/ L medium) than the media of $P$. gingivalis and $T$. forsythia $(13.5 \mathrm{gr} / \mathrm{L}$ medium) and, therefore, causing larger IZs. In comparison to the microdilution, the agar diffusion method requires larger amounts of material and at least 3 days for growing the anaerobic fastidious bacteria in order to well distinguish the inhibition zone from the surface growth. Additionally, the accommodation of one agar plate is no more than 5-6 spots of samples placed on the agar surface. Therefore, the current study preferred the use of the microdilution method as it deals with small amount of materials, enabling to compare extended diluted concentrations of more than one agent in the same time in one plate. In addition to these advantages, the incorporation of resazurin dye into the microdilution procedure adds further interest, peculiarity as it enables the colorimetric reading of the result after $34 \mathrm{hrs}$. of incubation. This could be simply achieved by tracing the dark blue color of resazurin in the wells containing the antimicrobial agents in each row and pointing the concentration of the agent in the last well with no color change as a MIC value (Figure- 7). This is of a great benefit when testing the susceptibility of fastidious anaerobic periodontal bacteria to reduce the time required in the agar diffusion method. It is an easy and accurate method to be followed as its results indicate a more realistic MIC concentration with no change of resazurin, whereas the presence of even few numbers of cells respiring at low levels leads to changes in the color. Thus, after adding the dye and in order to determine the MIC value, it is very important to compare the color of each well, to which the bacteria and the antibacterial reagent are added (column 1-10), with the color of both positive (column 11) and negative control (column 12). In the first row of the columns, the higher concentrations of olibanum made the color differ from both positive and negative control. Also, the color of resazurin differed 
from both controls in the higher concentrations of alum because of its acidic $\mathrm{pH}$. But, when the two materials were diluted, the color was more similar to the negative or positive controls.

Some other researchers reported similar notices about the reliable method in testing antimicrobial activity against anaerobic bacteria. The agar diffusion method was described as laborious, time consuming, requiring significant quantities of the test materials and, most importantly, causing confusion when dealing with low soluble materials which may affect the accuracy [3]. Also, in accordance to our study, the microdilution well plate- based method was preferred elsewhere $[4,20$, 21] as standardized, accurate, inexpensive and easy to perform. This overcomes the problems associated with low soluble tested materials and allows the detection of microbial growth in extremely small volumes of solution in microtitter plates. Also, upgrading this method by the addition of the redox indicator resazurin dye allows the visual reading of the results without the need to a spectrophotometer.

Resazurin reduction test has been used for decades to measure bacterial and yeast contamination of milk. Recently, it has been used by researchers in measuring cell viability tests to evaluate the biocompatibility of medical and dental materials as well as in cell proliferation and cytotoxicity assays to screen for new anti- proliferative substances against tumor cells, screen for E. coli K-12 strain for radiation sensitivity, and determine the MIC values of some biosurfactants against common bacterial species $[3,4,20]$. In the current study, the resazurin- based method proved validity to be followed in testing antimicrobials against the anaerobic fastidious periodontal bacteria as it offer accurate colorimetric- read results. Also, the superior benefit for this method lies in reducing the time usually required to obtain the results by agar diffusion methods. The current study used resazurin at $0.015 \mathrm{~g} /$ $100 \mathrm{ml}$ because higher concentrations of this dye may cause false negative results, as the active present bacteria will be unable to reduce the dye at higher concentrations [4].

Inhibitory effect of the four reagents on red complex pathogens: The above stated results of our study showed that all reagents under study exerted growth inhibitory activities against the three types of periodontal pathogens. The next discussion focuses on the results of the microdilution methods. By returning to the MIC values in Table- 3, it can be observed that olibanum and alum recorded MIC values of 1.4- 1.8 and $1.7-2.3 \mathrm{mg} / \mathrm{ml}$, respectively, which were higher than those for the two standards $(0.037-0.04 \mathrm{mg} / \mathrm{ml}$ for CHX and $0.0039 \mathrm{mg} / \mathrm{ml} \mathrm{CIP})$. This may be due to the use of these naturals as a crude extract while the standards are used as pure potent compounds. Also, the MIC values were slightly different between the three types of bacteria. In addition, it was seen that olibanum, alum, and CHX required higher MIC values to inhibit the growth of $P$. gingivalis than those required for the inhibition of $T$. denticola and $T$. forsythia, while CIP had the same MIC value on the three types of bacteria. To elucidate these differences, statistical analysis was useful to determine their significant meanings. According to the one sample $t$ - test, each agent had a significant inhibition within each bacterial type $(P$-value $<0.05)$, but ANOVA test showed no significant differences of this inhibition between bacterial groups $(P$ - value $>0.05)$. When comparing the effects of all agents by ANOVA test, it was shown that there were no significant differences between the effects of the reagents, except that of olibanum which significantly differed from the others and was the most effective, followed by CHX, CIP and alum.

CHX was reported to be the best local washing prescribed in the course of periodontitis treatment at a concentration of $120-200 \mathrm{mg} \%(0.12-0.2 \mathrm{w} / \mathrm{v} \%)$ [18]. The concentration of the commercial $\mathrm{CHX}$, which is equal to $1.2-2 \mathrm{mg} / \mathrm{ml}$, is much higher than the MIC value found in the current study (0.037- 0.04) $\mathrm{mg} / \mathrm{ml}$. CIP was reported to have the lowest MIC among several tested antibiotics commonly used in the treatment of periodontitis [5]. The prescribed oral dose of CIP is 250-500 mg twice daily, which attained its mean level in humans gingival crevicular fluid (GCF) at 0.00338 $0.00124 \mathrm{mg} / \mathrm{ml}$ after $2-7 \mathrm{hr}$., respectively, when the higher dose of $500 \mathrm{mg}$ was used [22]. This is lower than the MIC value recorded in the current study $(0.0039 \mathrm{mg} / \mathrm{ml})$, therefore, it is recommended for the periodic check of the MIC value of the common antibiotics.

The two natural materials, olibanum and alum, also exerted their validity in combating the periodontal pathogens. Hence, the statistical significant differences of growth inhibition continued to high extents of dilution. Olibanum is a safe and low cost herbal medicine which has been proved for the treatment of inflammatory and infectious diseases with various physiological properties, such as the immunomodulatory, anti-inflammatory, anti-cancer, anti-bacterial, and anti-fungal properties [23, 24]. The remarkable effects of olibanum are attributed to the multifunctional constituents present in 
the crude extract which was analyzed by previous studies. It consists of essential oils, gum, and terpenoids. The boswellic acids in the terpenoid portion were reported as the active constituents for anti- inflammatory effects. Resins fraction was reported to destroy the microbial cell wall and stop protein synthesis in Strep. salivarius, S. aureus, and B. megaterium. Water soluble gum has a sticky character which stops the reactions between substances [25]. Keto-ß-boswellic acid was reported to distort the cell membrane structure and disrupt the permeability barrier of microbial membrane structures. Olibanum was also reported to be a DNA intercalator and an inhibitor of bacterial DNA synthesis through topoisomerase inhibition [24].

The current study used the crude aqueous extract of olibanum and recorded the MIC values of 1.8 $\mathrm{mg} / \mathrm{ml}$ against $P$. gingivalis and $1.4 \mathrm{mg} / \mathrm{ml}$ against $T$. forsythia and $T$. denticola. In comparison, earlier reports recorded a value of $8 \mathrm{mg} / \mathrm{ml}$ or $25 \mathrm{mg} / \mathrm{ml}$ of the crude extracts against G-ve and G+ ve isolates $[9,24]$. Other studies tested the extracted active molecules from olibanum. A previous study [26] showed that the extracted active compounds of the essential oils of olibanum at $0.0066 \mathrm{mg} / \mathrm{ml}$ showed inhibitory activities against $E$. coli and three strains of $S$. aureus, with the greatest activity being recorded against $P$. aeruginosa and against Candida albicans, whilst it had no inhibitory activity against methicillin resistance Staphylococcus aureus (MRSA) strain. Oleogum resin extracts of four oils were reported in other study [27] to have antibacterial activities against the clinical isolates of $B$. subtilis, $M$. luteus, S. aureus, $K$. pneumoniae, and E. aerogenes, while those of $P$. aeruginosa, E. coli, and $P$. vulgaris were resistant. The oil fraction in an earlier study [28] showed moderate to poor activities against the reference strain of $S$. aureus. In addition, the four oil extracts in another study [29] reported growth inhibition against several Gram-positive strains, with strongest activities (lowest MIC $1.87 \mathrm{mg} / \mathrm{ml}$ ) against $S$. aureus and B. subtilis, while G-ve bacteria were less susceptible with no activity against the fungus $C$. albicans. The presence of limonene in the oil fraction was responsible for the antifungal activity against $C$. albicans [26], whereas the absence of this component was responsible for inactivity against the yeast [29]. Other authors [30] tested several isolated molecules of the boswillic acid against G-ve and G+ve bacteria. They reported that G-ve bacteria were more resistant, which may be attributed to the presence of the lipophilic outer membrane. The most active compound was acetyl keto $\beta$ - boswellic acid with a lowest MIC of $0.002 \mathrm{mg} / \mathrm{ml}$.

In the context of comparing the results of alum with other studies, the activities of $2.5 \mathrm{mg} / \mathrm{ml}$ to reduce the CFU of S. aureus, S. epidermidis, E. coli, Klebsiella pneumonia, and S. mutans were reported with significant differences compared with the standard cefotaxime [31]. Another report of 15 $\mathrm{mg} / \mathrm{ml}$ of alum showed a significant inhibition compared to ofloxacin against Proteus sp., E. coli, B. subtilis and Klebsiella sp. [14]. The mechanism of bactericidal effect of alum is not clear. Some hypotheses attribute the antibacterial effect to the reduction in acidity when dissolved in water, forming sulfuric acid which causes protein precipitation or deleterious effects on bacterial cell wall. Histological studies verify the safety of alum salt for mammalian cells as it cannot be directly absorbed due to its negatively charged molecule. Concentrations of $3.0 \%$ have been considered noncytotoxic, but higher concentrations might cause nephrotoxicity and intestinal bleeding $[11,14]$.

Conclusion: The current study preferred the use of the resazurin- based microdilution method which shows a comprehensive application. Olibanum and alum had useful benefits as safe natural materials to control the chronic periodontitis by reducing the growth of the causative agents red complex group. From our results and the previously outlined ones, we can conclude that the MIC values recoded from the extracted active molecules were much lower than those from the aqueous crude. It is difficult to attribute the antimicrobial activities to one single component as the extracted fraction had an antimicrobial effect against a microbial type in one study but no effect in others. Some extracted molecules were having less activity against G-ve bacteria. Previous studies proved the antimicrobial activities of crude olibanum extract against $\mathrm{G}$-ve and $\mathrm{G}+\mathrm{ve}$ aerobes. The current study proved the activity of the aqueous crude extract against the anaerobic G-ve red complex periodontal pathogens.

\section{References}

1. Sarker, S., Nahar, L. and Kumarasamy, Y. 2007. Microtitre plate- based antibacterial assay incorporating resazurin as an indicator of cell growth, and its application in the in vitro antibacterial screening of phytochemicals. Methods, 42: 321-324.

2. Brook, I., Wexler, H. and Goldsteinc, E. 2013. Antianaerobic antimicrobials: spectrum and susceptibility testing. $C M R, 26: 526-546$. 
3. Hudman, D. and Sargentini, N. 2013. Resazurin-based assay for screening bacteria for radiation sensitivity. SpringerPlus, 2:55-60.

4. Elshikh, M., Ahmed, S., Funston, S., Dunlop, P., McGae, M., Marchant, R. and Banat, I. 2016. Resazurin- based 96-well plate microdilution method for the determination of minimum inhibitory concentration of biosurfactants. Biotechnol Lett, 38: 1015-1019.

5. Muhammad, S. and Al-Rawi, A. 2011. Detection of the Efficacy of some Transport, Isolation and Culture Media for Oral Treponema Isolated from Periodontitis. Raf. J. Sci., 22: 49- 67.

6. Schmitt, D., O'Dee, D., Cowan, B., Birch, J., Mazzella, L., Nau, G. and Horzempa, J. 2013. The use of resazurin as a novel antimicrobial agent against Francisella tularensis. Frontiers in Cellular and Infection Microbiology, 3: 1-6.

7. Bedran, T., Oliveira, G., Spolidorio, L., Cirelli, J. and Spolidorio, D. 2016. Comparison of two different methods for detecting periodontal pathogenic bacteria. Braz. J. Oral Sci, 15: 166-172.

8. Bogdanovska, L., Kukeska, S., Popovska, M., Petkovska, R. and Goracinova, K. 2012. Therapeutic strategies in the treatment of periodontitis. Mac Pharm Bull, 58: 1-12.

9. Ismail, S., Aluru, S., Sambasivarao, K. and Matcha, B. 2014. Antimicrobial activity of frankincense of Boswellia serrata. Int. J. Curr. App. Sci., 3: 1095-1101.

10. Nawab Al- Deen, F. 2017. Evaluation of antibacterial activity of various solvents extracts of Annona squamosal fruit. Iraqi Journal of Science, 58: 2301- 2308.

11. Ali, Z. 2018. Synergistic antibacterial interaction between alum and antibiotics on some microorganism. Sci J Med Res, 2: 47- 51.

12. Salman, A., Amer, Z. and Abud- Rahman, E. 2017. Bacteriological study of Pseudomonas aeruginosa isolated from different infections and study antimicrobial activity of plant extract Solanum nigrum against it. Iraqi Journal of Science, 58: 2278- 2284.

13. Hussain, H., Al-Harrasi, A., Al-Rawahi, A. and Hussain, J. 2013. Chemistry and Biology of Essential Oils of Genus Boswellia. Review Article, Evidence-Based Complementary and Alternative Medicine, 12:1-12

14. Amadi, L. and Ngerebara, N. 2017. Susceptibility Profiles of alum on bacteria isolated from shellfish bivalve oyster. Int. J. Curr. Microbiol. App. Sci, 6: 941-947.

15. Heyman, L., Houri-Haddad, Y., Heyman, S., Ginsburg, I., Gleitman, Y. and Feuerstein, O. 2017. Combined antioxidant effects of Neem extract, bacteria, red blood cells and Lysozyme: possible relation to periodontal disease. $B M C, 17: 2-8$.

16. Kotsilkov, K., Popova, C., Boyanvova. L., Setchanova, L. and Mitov, I. 2015. Comparison of culture method and real-time PCR for detection of putative periodontopathogenic bacteria in deep periodontal pocket. Biotechnology and biotechnological equipment, 29: 996-1002.

17. Dashper, S., O'Brien- Simpson, N., Liu, S., Paolini, R., Mitchell, H., Walsh, K., D'Cruze, T., Hoffmann, B., Catmull, D., Zhu, Y. and Reynold, E. 2014. Oxantel disrupt polymicrobial biofilm development of periodontal pathogens. J.A.A.C. 58: 378-385.

18. Wang, H. and Ren, D. 2017. Controlling Streptococcus mutans and Staphylococcus aureus biofilm with direct current and chlorhexidine. AMB Express, 7: 204- 212.

19. Montevecchi, M., Dorigo, A., Cricca, M. and Checchi, L. 2013. Comparison of the antibacterial activity of an ozonated oil with chlorhexidine digluconate and povidone-iodine. A disk diffusion test. New microbiologica, 36: 289- 302.

20. Borra, R., Lotufo, M., Gagioti, S., Barros, F. and Andrade, P. 2009. A simple method to measure cell viability in proliferation and cytotoxicity assays. Braz. Oral Res., 23:255-262.

21. Tye, K., Gan, S., Lim, S., Tan, S., ou-Eei, Chen, C. and Phang, S. 2016. Comparison of visual observation and emission intensity of resazurin for antimicrobial properties of hexane, dichloromethane, methanol and water extracts from a brown alga, Turbinaria ornate. Microbiology, parasitology and virology research article, Cogent Biology, 2: 1-14.

22. Dincel, A., Caglayan, F. and Bozkurt, A. 2005. Determination of ciprofloxacin in human gingival crevicular fluid by high performance liquid chromatography. ACTA Chroma., 15: 308- 314.

23. Langmead, L. and Rampton, D. 2006. Complementary and alternative therapies for inflammatory bowel disease. Review article, Alimentary Pharmacology and Therapeutics, 23: 341- 349.

24. Al-kuraishy, H., Al-gareeb, A., Ashoor, A. and Al-windy, S. 2012. Modifying effects of Boswellia carteri on clarithromycine action: In vitro antibacterial study against common sensitive bacterial strains. IJDDR, 4: 155-162. 
25. Sabra, S. and Al-Masoudi, L. 2014. The Effect of Using Frankincense (Boswellia sacra) Chewing Gum on the Microbial Contents of Buccal/Oral Cavity, Taif, KSA. J. Den. Med. Sciences, 13: 7782.

26. Camarda, L., Dayton, T., Di Stefano, V., Pitonzo, R. and Schillaci, D. 2007. Chemical composition and antimicrobial activity of some oleogum resin essential oils from Boswellia spp. (Burseraceae). Annali di Chimica, 97: 837- 844.

27. Al-Saidi, S., Rameshkumar, K., Hisham, A., Sivakumar, N. and Al- Kindy, S. 2012. Composition and antibacterial activity of the essential oils of four commercial grades of Omani Luban, the oleogum resin of Boswellia sacra. Chemistry and Biodiversity, 9: 615- 624.

28. Van, S., Kamatou, F. and Viljoen, A. 2010. Volatile composition and antimicrobial activity of twenty commercial frankincense essential oil samples. South African Journal of Botany, 76: 686691.

29. Mothana, R., Hasson, S., Schultze, W., Mowitz, A. and Lindequist, U. 2011. Phytochemical composition and in vitro antimicrobial and antioxidant activities of essential oils of three endemic Soqotraen Boswellia species. Food Chemistry, 126: 1149- 1154.

30. Raja, A., Ali, F., Khan, I., Shawl, A., Arora, D., Shah, B. and Taneja, S. 2011. Antistaphylococcal and biofilm inhibitory activities of acetyl-11- keto- $\beta$ - boswellic acid from Boswellia serrate. BMC microbiology, 11: 54- 62.

31. Ali, A., Hamiduddin, and Zaigham, M. 2017. Shibb-E-Yamani (Alum) a unique drug and its utilization in Unani medicine: a physiological and pharmacological review. Int $J$ Res Ayurveda Pharm, 8: 17-22. 